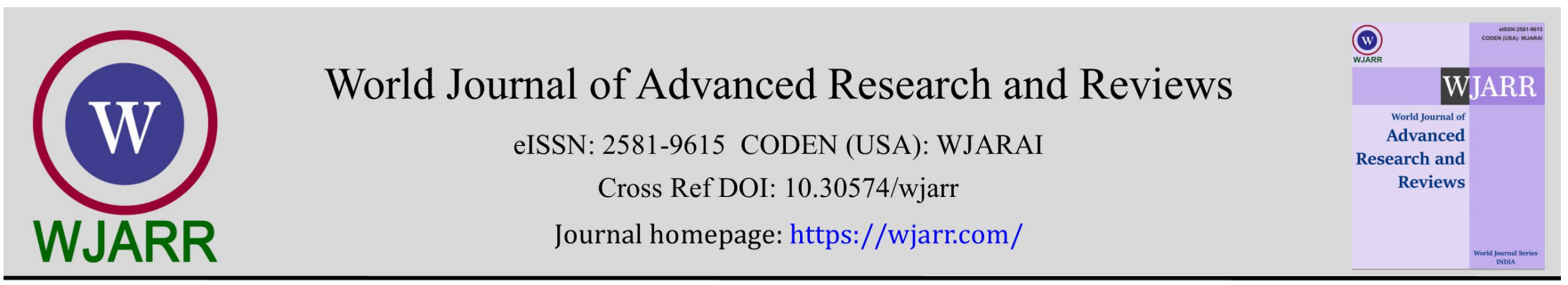

(RESEARCH ARTICLE)

\title{
Illustration of the connection between the vastus medialis obliquus muscle and the superficial medial collateral ligament of the knee: A human knee cadaveric study
}

\author{
Mohamed Hamid Awadelseid * \\ Department of Orthopedic Excellence Trauma Centre- Khartoum, Sudan.
}

World Journal of Advanced Research and Reviews, 2021, 12(01), 431-438

Publication history: Received on 14 September 2021; revised on 23 October 2021; accepted on 25 October 2021

Article DOI: https://doi.org/10.30574/wjarr.2021.12.1.0531

\begin{abstract}
Background: While the anatomy of the medial part of the knee has been extensively described, the muscular connections to the superficial medial collateral ligament (SMCL) have not been sufficiently studied. The purpose of this study is to describe the anatomy of the musculo-ligamentous connection between the sMCL and the Vastus Medialis Obliquus muscle (VMO), and to describe its anatomy.
\end{abstract}

Methods: Six Human Cadaveric knees were used in this study. Donors were 4 males and 2 females with a mean age of 49 years old. Dissection was performed in fixed knee extension and directed to show the area of the proximal attachment of the sMCL.

Results: A musculo ligamentous connection between the distal portion of the Vastus medialis Obliquus muscle and the sMCL has been identified in our entire specimens. The mean mid substance width of this connection was 9.75 (8.7 -10.8) $\mathrm{mm}$, the mean length was 29.3 (22.2-36.4) $\mathrm{mm}$ and the mean thickness was $1.3(0.9-1.7) \mathrm{mm}$.

Conclusion: The proximal femoral attachment of the SMCL is directly connected to the distal end of VMO. This connection may show that the sMCL can possibly assist in the dynamic stabilization of the knee during extension against valgus stress, through its tension by the contracted VMO muscle.

Keywords: Superficial Medial collateral ligament; Anatomy; Human Knee

\section{Introduction}

The medial knee stabilizers are the most frequently injured ligaments of the knee. Also, the medial collateral ligament (MCL) is injured in at least $42 \%$ of knees with ligamentous injuries [1], being much more common inyounger population and in males more than females [2]. Secondary to a mechanism of injury including forceful knee valgus stress and/ or external rotation, and with increased prevalence of injury during certain athletic activities such as soccer and skiing [3], MCL injury can occur in isolation, orin association with anterior cruciate ligament injury, and medial meniscustears [4]. Classically, most of isolated MCL injuries could heal with conservative management, but high-grade or chronic injury may lead to chronic knee instability [5] and may necessitate surgical repair orre construction. Complete knowledge of the anatomy of the MCL could help int he management of medial side knee injuries, whether a conservative or surgical intervention was planned, to obtain favorable clinical results.

\footnotetext{
${ }^{*}$ Corresponding author: Mohamed Hamid Awadelseid

Department of Orthopedic Excellence Trauma Centre- Khartoum, Sudan.
}

Copyright $(2021$ Author(s) retain the copyright of this article. This article is published under the terms of the Creative Commons Attribution Liscense 4.0. 


\subsection{Anatomical considerations}

The medial side of the knee has been classically described as being three layered [6]. The first layer, being most superficial, includes the Sartorius muscle and its investing fascia. The second layer includes the sMCL, posterioroblique ligament(POL), medial patellofemoral ligament (MPFL) and the musculo-tendinous attachments of the Semimembranosus. The third and deepest layer consists of the deep MCL and the joint capsule.

The two components of the MCL (superficial and deep) lie in the middle and deepest layers respectively and are separated by a bursa [8].

The medial side of the knee contains both dynamic and static stabilizers that contribute to the resistance of valgus and rotational forces. Static stabilizers of the medial knee include the sMCL, the deep MCL and the POL[9]. The medial dynamic stabilizers are the Semimembranosus complex, the quadriceps, and the pes anserinus. The MCL is the main stabilizer against valgus stress at lower flexion angles, and is known to provide 78\%of the restraining force against valgus injury at 25 degrees of knee flexion, and $57 \%$ of the restraining forces against valgus stress in full knee extension. The POL, ACL and posteromedial capsule aid to provide most of the remaining restraint in full extension[10].

The sMCL origin is a round to oval depression posterior and 65 proximal to the femoral epicondyle. Proximal and posterior to the SMCL origin, the superficial and central arms of the POL are attached to bone near to the medial gastrocnemius tubercle. The superficial and central arms of the POLrun distally to insert into the distal tibial expansion of the semimembranosus and its tibial attachment, the posteromedial aspect of the medial meniscus, the meniscotibial portion of the posteromedial capsule, and the posteromedial part of the tibia [11]. The medial patellofemoral ligament is afibrous structure that extends from a broad area along the supero media laspect of the medial border of the patella and also from terminal end of the VMO muscle, and run medially and posteriorly to insert into the bone and soft tissue between the medial femoral epicondyle and the femoral attachments of the adductor magnus tendon into the adductor tubercle offemur [10].

In his description of the anatomy of the knee, Muller W [13] noted thepresence of ligamentous connection between the vastus medialis muscle and its fascial covering, and the area of MCL origin around the medial femoral epicondyle (MFE). Muller W [13] stated that on the femur these ligamentous collagenous fibers are in close association with the normal ligament and even appear to be blended with it over part of their course, and they terminate directly or indirectly to the vastus medialis muscle, and aids in active tension of MCL during motion. Based on that, Indlicato P [7] has also mentioned that the MCL is dynamized by the contraction of the Vastus Medialis during active extension through this connection. However; the anatomy of this connection has not been described in any further literature. This Cadaveric study aims to identify and illustrate the anatomy of this connection.

\section{Material and methods}

\subsection{Cadaveric Knees}

Six frozen and unpaired human cadaveric knees were utilized for this study. All the cadaveric specimens were available in our medical schools for researchers. The specimens were 3 right and 3 left knees without known pre existing knee surgery, belong to 4 males and 2 females. The mean age of the donors was 49 years (range 47 to 52). Each specimen was frozen at $-20^{\circ} \mathrm{C}$ and thawed overnight prior to dissection. Each knee was transected $\sim 35 \mathrm{~cm}$ above and below the joint line. The specimen was then aligned in a full extension position which was maintained throughout the dissection and measurements.

\subsection{Anatomical dissection}

First, the skin and subcutaneous tissue were dissected. The pes anserinustendons (sartorius, gracilis, and semitendinosus tendon) and the MPFL were detached from their distal attachments during dissection. Identification of thes MCL in the middle layer of the medial knee compartment is done. Thes MCL origin around the medial epicondyle was identified and carefully raised and followed proximally to the direction of the vastus medialis. At this stage, a Musculo ligamentous connection between the SMCL and VM was identified and raised carefully off the bone. Measurements were performed using the digital caliber ruler device (range 0-150 mm, precision 0.02mm).

\section{Results}

\subsection{Musculo-Ligamentous connection between the VMO and SMCL}

In all our specimens, we have dissected and proven a musculo-ligamentous connection, closely related to the underlying bone that extends between theVMO muscle and the uppermost fibers and anterior border of sMCL at its origin around the medial epicondyle. (Fig 1,2,3-6).This connection consists of mainly ligamentous and few muscle fibers. It originates 
from the posterior border of distal end of theVMO muscle proper and the fascia covering it above the medial epicondyle. Also it has contribution from the adductor fascia and tendon. Then it extends distally, being closely related to the underlying bone, to cover the superior most portion of the medial femoral epicondyle, blending with the most superficial fibers of superior and anterior border of sMCL fibers in a fan shaped manner. (Fig 1, 2, 4, 5).

The shape and size of this connection varies from a specimen to another, depending on the muscle mass and the length of the limb, being more defined in specimens with good muscle mass. The length of the ligamentous portion of this connection from its origin at the VM to its end at the sMCL ranged from 22.2 to 36.4 (average 29.3) mm. We have divided it into thirds and measured the antero -posterior width for each one third. The average widths were 12.6-15.7 (14.1) $\mathrm{mm}, 14,1-17.5$ (15.8) $\mathrm{mm}$ and $8.7-10.8$ (9.75) $\mathrm{mm}$ for the proximal, distal and middle parts respectively. The thickness of this connection was not uniform, being thicker and broader proximally then tapering distally till it ends in the sMCL. In our specimens, the average mid substance thickness was about 0.9-1.7 (1.3) mm. The ligamentous fibers measurements in this connection are summarized in table 1.

Table 1 Measurements of sMCL-VMO connection: Range, (Average)

\begin{tabular}{|c|c|c|c|c|c|}
\hline $\begin{array}{c}\text { Proximal } \\
\text { insertion } \\
\text { width }\end{array}$ & $\begin{array}{c}\text { Distal } \\
\text { insertion } \\
\text { width }\end{array}$ & $\begin{array}{c}\text { Mid } \\
\text { substance } \\
\text { Width }\end{array}$ & Length & $\begin{array}{c}\text { Mid } \\
\text { substance } \\
\text { thickness }\end{array}$ & site \\
\hline $\begin{array}{c}12.6-15.7 \\
(14.1) \mathrm{mm}\end{array}$ & $\begin{array}{c}14.1-17.5 \\
(15.8) \mathrm{mm}\end{array}$ & $\begin{array}{c}8.7-10.8 \\
(9.75) \mathrm{mm}\end{array}$ & $\begin{array}{c}22.2-36.4 \\
(29.3) \mathrm{mm}\end{array}$ & $\begin{array}{c}0.9-1.7 \\
(1.3) \mathrm{mm}\end{array}$ & Measurement \\
\hline
\end{tabular}

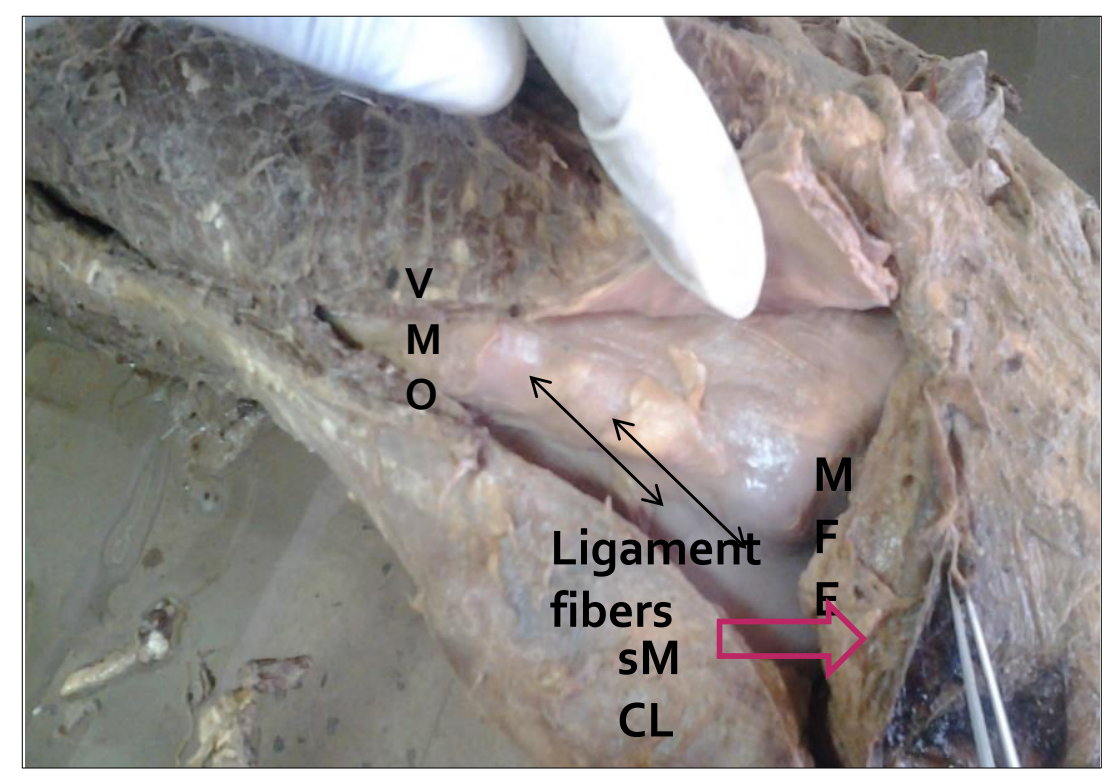

Figure 1 Cadaveric exposure showing the Medial Femoral Epicondyle (ME), the Vastus Medialis muscle (VM), the superficial Medial Collateral ligament origin (sMCL). The soft tissue structures were not elevated off the bone. Note the bright ligamentous fibers that originate from the undersurface and posterior border of VMO muscle and the fascia covering its tendon before joining the medial border of patella,). Then it extends distally, to cover the medial femoral epicondyle (ME), and then to blend with the most superficial fibers of superior and anterior border of sMCL fibers at the level of their origin from the Medial epicondyle 


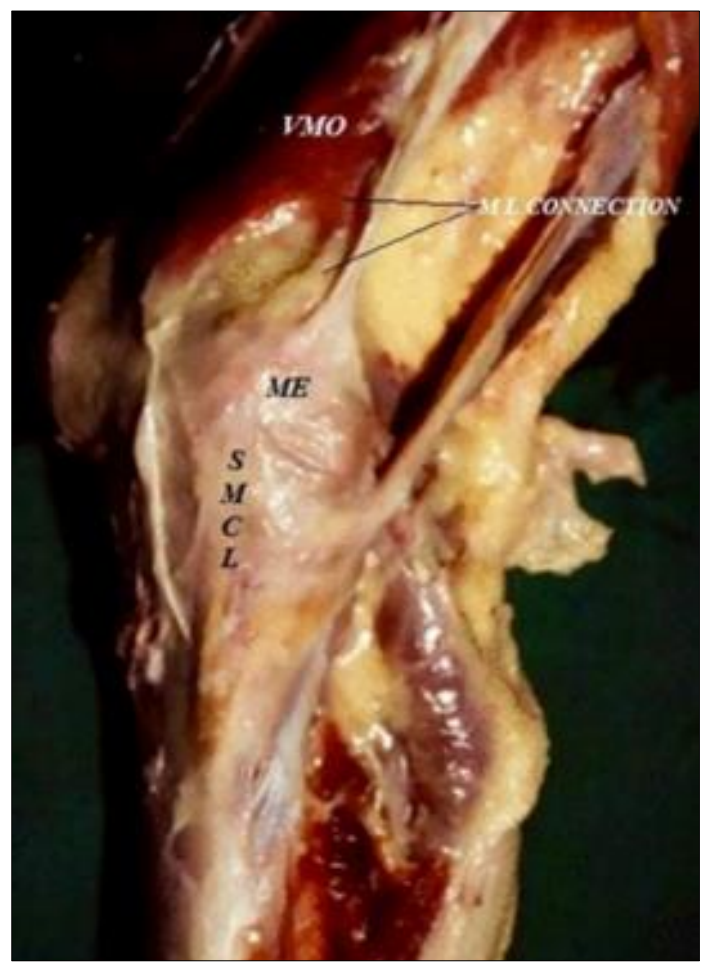

Figure 2 Cadaveric exposure of the medial knee shows the Musculo Ligamentous connection between the Vastus Medialis Obliquus (VMO) and the origin of the Superficial Medial Collateral Ligament (s MCL) from the Medial Epicondyle (ME). Note the contribution of the fascial covering VMO tendon to the connecting fibers. Reprinted with permission from Michel Bonnin: The Knee Joint, Surgical techniques and strategies, Springer - Verlag France, Paris, $2012[3]$

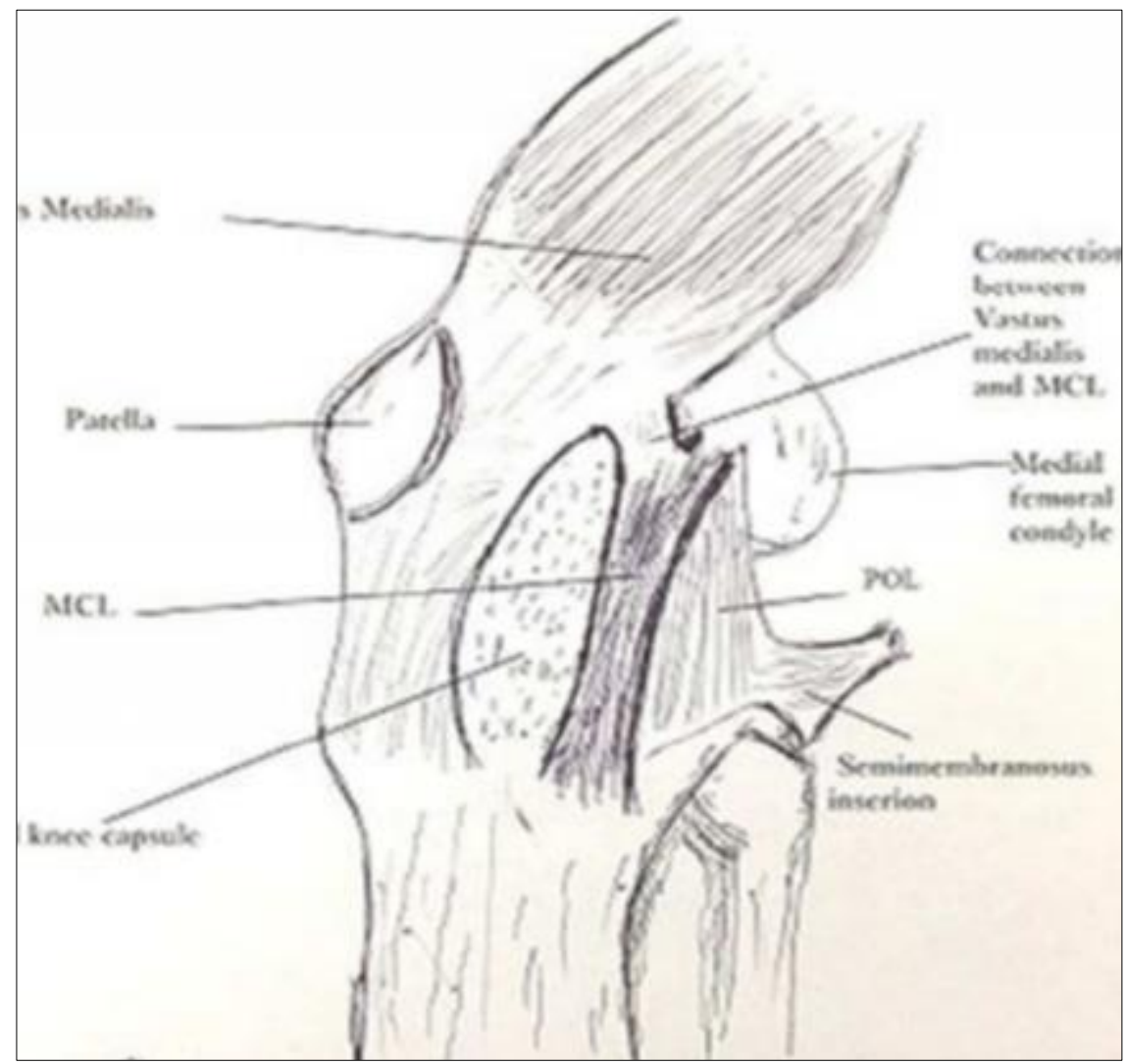

Figure 3 Simplified illustration of the connecting sleeve between the VMO and s MCL 


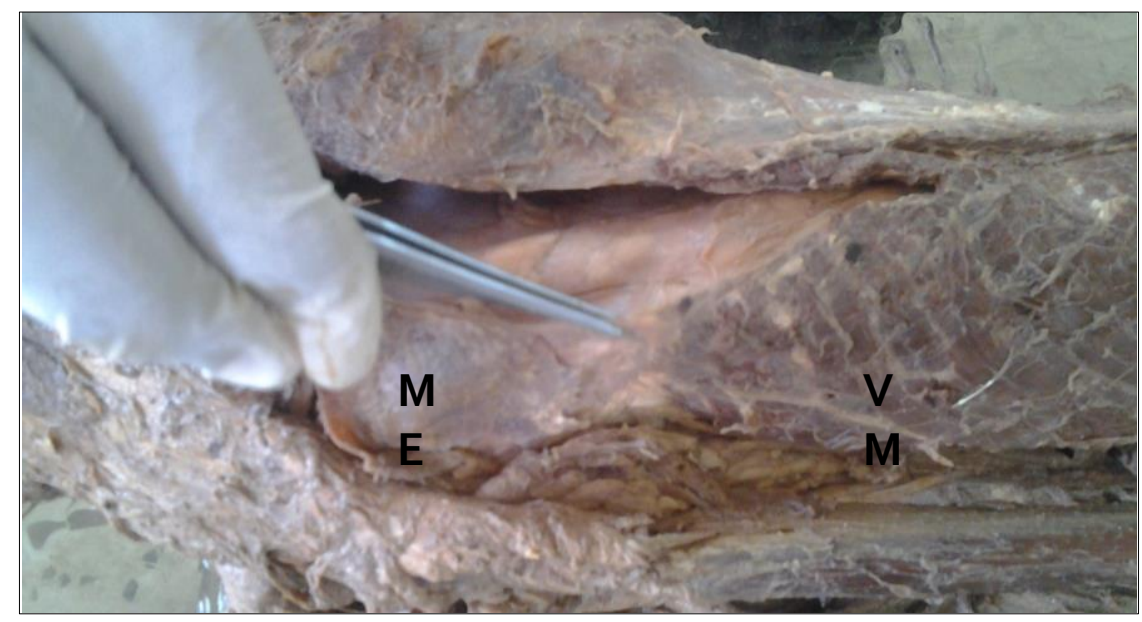

Figure 4 Cadaveric knee sample. Note the forceps pointed musculo ligamentous tissue that extends from the lower fibers of VMO to the area of SMCL origin from the medial epicondyle
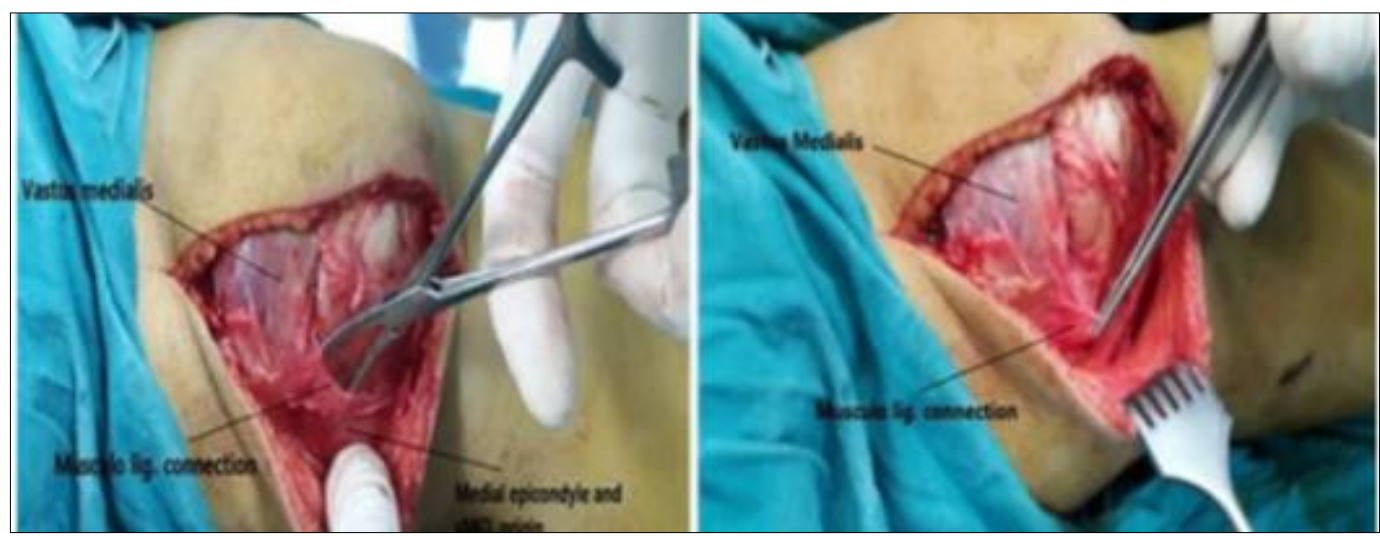

Figure 5 Clinical photos, illustrating the connection between the s MCL and VMO, dissection done during a surgery for recurrent patellar dislocation

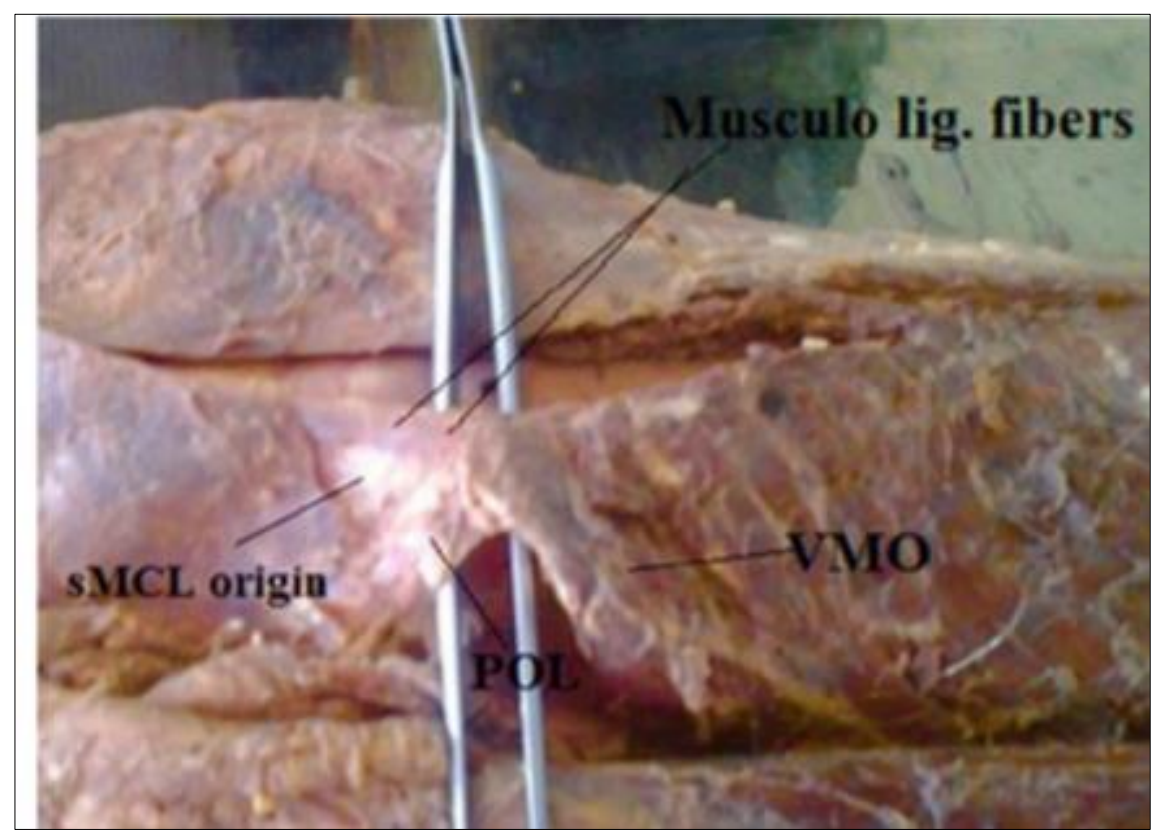

Figure 6 Cadaveric knee sample. Note the elevated musculo ligamentous connection from the distal end of Vastus Medialis to the sMCL origin from the medial epicondyle 


\section{Discussion}

This study aims at determining the morphology of the connecting sleeve between the sMCL and the VMO. To our knowledge, there are no reports quantifying this connection except a short note by Muller W [13], who pointed out the existence of ligamentous collagenous fibers that can be traced beyond the ideal lines of the MCL origin. Muller W [13] mentioned that these fibers dynamize the MCL through its attachment to the Vastus Medialis and adductors, and stated that the SMCL fibers are protected from possible overstretching through its attachment to a dynamic, mobile structures as the muscle. In our study, we found that this connection to originate as muscular fibers from the VMO, and takes contributions from the adductor tendon and its covering fascia, to run distally as thin ligamentous fibers, closely related to the underlying bone to blend with the superior and anterior borders of the MCL at its origin from the medial epicondyle (Fig 1, 2, 4, 6).This connection may be overlooked due to many reasons; the first is that it arises from a small portion from the posterior border of most distal end of the VMO, and may be easily unnoticed in specimen with thin VMO muscle 171 mass. Also, this connection could be closely related to the underlying bone and often needs to be elevated carefully to expose it.

As mentioned in literature, the POL portion of the MCL was classically believed to assess in the dynamic stability of the knee during knee flexion, through its connection to the Semimembranosus tendon [16], while the sMCL itself was classically stated to be a static knee stabilizer. The presence of a direct connection between the sMCL and VMO may change the concept about the pure static function of the sMCL. Moreover, we found that in specimens with a well-defined VMO muscle mass, and subsequently more defined connecting fibers; the anterior portion of the s MCL which receives the insertion of this connection is more prominent and thicker than the rest of the s MCL. That's why we believe that the anterior portion of the s MCL is adynamized portion through its connection to the active structures. Injuries to knee ligaments are very common. It has been estimated that the incidence could be at 2/1000 people per year in the general population witha much higher incidence in those who are involved in sports activities. Ninety percent of knee ligament injuries involve the anterior cruciate ligament (ACL) and the medial collateral ligament [12]. Basic science and clinical studies have revealed that a ruptured MCL can heal spontaneously [16], however, laboratory studies have shown that its ultra-structure and biochemical composition may remain significantly changed [14].

Furthermore, the mechanical properties of the ligament substance remain substantially inferior to those of normal ligaments even after years of remodeling [11]. Thus, there has been a tremendous need for knowledge regarding ligament anatomy to better understand ligament injuries, healingand remodeling in hope to develop new and improved treatment strategies.

The limitless ability of the dynamized portion of the ligament to best rengthened by training may lead to increased ability to adapt higher performance, and could explain why the well-trained athletes suffer fewer injuries than fewer ones, and why less lower extremity muscle mass was associated with greater laxity and less stiffness in frontal and transverse planes. This also may contribute to the disproportionally higher laxities andreduced stiffnesses observed in females in these planes more than males, and why instabilities are particularly disabling in individuals with poor muscular strength $[13,18]$. Muller $\mathrm{W}[13]$ postulated that flail knee after poliomyelitis is a consequence of laxity of the "rigid" ligaments due to chronic overstretching, when they can no longer be protected from excessive stress bya $\mathrm{n}$ actively dynamized ligament component Shultz et al [19], concluded that transverse plane laxity and stiffness, or varus and valgus instabilities, may be modifiable through strength training interventions that promote changes in muscle characteristics (e.g. muscle cross-sectional area) thus contribute to more dynamic joint stability during sport activity. Through its connections to the static structures, a good muscle function could add considerable dynamic properties to the static structures and increase its ability to withstand stresses. Although, the biomechanical value of such small connection between the VMO and the sMCL might be less likely to significantly affect the knee stability, we believe that proper rehabilitation program for the medial knee instability should focus on increasing the VMO muscle mass and hence increasing the efficiency both the dynamic and static knee stabilizers functions. We admit several limitation of this study. First, is being only a descriptive study, while a biomechanical assessment of these findings would be most appropriate for investigating its value. Second, more specimens are needed to properly assessing the size of such connection. We believe that more specimens and biomechanical assessment would be most appropriate to illustrate such anatomical connection. In summary, this study describes the anatomy of musculo ligamentous connection between the VMO and the SMCL in human knee specimens. Morework is needed to quantitatively determine the bio mechanical value of this connection in dynamization of the MCL during various degrees of knee movement. 


\section{Conclusion}

The proximal femoral attachment of the SMCL is directly connected to the distal end of VMO. This connection may show that the SMCL can possibly assist in the dynamic stabilization of the knee during extension against valgus stress, through its tension by the contracted VMO muscle.

\section{Compliance with ethical standards}

\section{Acknowledgments}

Thank to prof. Kh. Emara and Elnelain University Anatomy Department who contributed by any mean to the success of this study.

\section{Conflict of interest}

None to declare.

\section{Statement of informed consent}

All the cadaveric specimens were available in our medical schools for researchers and under full authorization from our hospitals board and health authorities. Approval of this study was obtained from our institutional review board.

\section{References}

[1] Abbott LC, Saunders JBDM, Bost FC, Anderson CE. Injuries to the ligaments of the knee joint. J Bone Joint Surg Am. 1944; 26(3):503-521.

[2] Bollen S. Epidemiology of knee injuries: diagnosis and triage. Br J Sports Med. 2000; 34(3): 227-228.

[3] Bonnin M, Amendola A, Bellemans J, MacDonald S, Menetrey J. The Knee Joint, Surgical techniques and strategies, Springer - VerlagFrance, Paris. 2012; 3: 24.

[4] Daniel DM, Pedowitz RA, O'Connor JJ, Akeson WH Daniel's knee injuries: ligament and cartilage: structure, function, injury, and repair. Lippincott Williams \& Wilkins, New York. 2003.

[5] Frank C, Woo SLY, Amiel D, Harwood F, Gomez M, Akeson W. Medial collateral ligament healing. A multidisciplinary assessment in rabbits. Am J Sports Med. 1983; 11(6): 379-3 89.

[6] Grood ES, Noyes FR, Butler DL et al. Ligamentous and capsular restraints preventing straight medial and lateral laxity in intact human cadaver knees. J Bone Joint Surg Am. 1981; 63: 1257-1269.

[7] Indelicato PA. Isolated Medial Collateral Ligament Injuries in the Knee, J Am AcadOrthop Surg. 1995; 3: 9-14.

[8] LaPrade RF, Engebretsen AH, Ly TV, Johansen S, Wentorf FA, Engebretsen L. The anatomy of the medial part of the knee. J Bone Joint Surg Am. 2007;89:2000-10.

[9] Last RJ: Some anatomical details of the knee joint. J Bone Joint Surg Br. 1948 Nov;30B(4):683-8.

[10] Liu F, Yue B, Gadikota HR, Kozanek M, Liu W, Gill TJ, Rubash HE, Li G. Morphology of the medial collateral ligament of the knee. JOrthopSurg Res. 2010; 5:69.

[11] Loitz-Ramage BJ, Frank CB, Shrive NG. Injury 276 size affects long-term strength of the rabbit medial collateral ligament. ClinOrthopRelat Res.1997; 337: 272-280.

[12] Myasaka KC, Daniel D, Stone ML, Hirschmann P. The incidence of knee ligament injuries in the general population. Am J Knee Surg.1991;4:3-7.

[13] Müller W. The Knee: Form, Function, and Ligament Reconstruction. New York: Springer-Verlag. 1983.

[14] Niyibizi C, Kavalkovich K, Yamaji T, Woo SLY. Type V collagen is increased during rabbit medial collateral ligament healing. Knee SurgSports Traumatol Arthrosc. 2000; 8(5): 281-285.

[15] O'Donoghue DH. Surgical treatment of fresh injuries to the major ligaments of the knee. J Bone Joint Surg Am 1950; $32(A: 4): 721-738$. 
[16] Phisitkul P, James SL,Wolf BR, Amendola A. MCL injuries of the knee: Current concepts review. The Iowa Orthopaedic Journal 2006;26:77-90.

[17] Warren LF, Marshall JL. The supporting structures and layers of the medial side of the knee: an anatomical analysis. J Bone Joint Surg Am. 1979 Jan;61(1):56-62.

[18] Wijdicks CA, Griffith CJ, Johansen S, Engebretsen L, LaPrade RF. Injuries to the medial collateral ligament and associated medial structures of the knee. J Bone Joint Surg Am. 2010; 92(5):1266- 1280.

[19] Shultz SJ, Pye ML, Montgomery MM, Schmitz RJ. Associations between lower extremity muscle mass and multiplanar knee laxity and stiffness: a potential explanation for sex differences in frontal and transverse plane knee laxity. Am J Sports Med.2012 Dec; 40(12):2836-44. 